Research Article

Yuan-Xia Fu*, Li Wang, and Li Xu

\title{
Electronic temperature characteristics of laser-induced Fe plasma in fruits
}

https://doi.org/10.1515/phys-2020-0005

Received Oct 24, 2019; accepted Dec 04, 2019

\begin{abstract}
Fruit is not only delicious, but it also contains iron, potassium, magnesium, and other microelements necessary for the human body. Banana, pineapple and kiwifruit were selected as samples for testing. A laser with a wavelength of $532 \mathrm{~nm}$ was focused on the samples' surface to generate a plasma. The emission spectrum of the atoms and ions in the plasma were collected by optical receivers, and the optimal condition was confirmed by analyzing the signal-to-noise ratio. The electronic temperature characteristics of the Fe plasma were studied under optimal experimental conditions. The maximum electron temperature of the Fe plasma occurred after a time delay of $300 \mathrm{~ns}$ in bananas, after $400 \mathrm{~ns}$ in pineapples and after $400 \mathrm{~ns}$ in kiwifruit. The electronic temperature of the Fe plasma increased with the time delay before the maximum point was reached. However, the temperature decreased after the maximum point was reached. At the beginning of the decline, the plasma decreased rapidly, but later decreased slowly. The range of the variation of the electron temperature of the Fe plasma in bananas, pineapples, and kiwifruits was 12388.29-19958.3 K, 11994.21-16856.4 K, and 13388.2-19607.32 K, respectively.
\end{abstract}

Keywords: laser-induced; fruit; spectrum; plasma; electron temperature

\section{Introduction}

Laser-induced plasma spectroscopy uses laser pulses as the energy sources and lenses to focus the laser on the surface of the sample [1]. The sample in the focus area

\footnotetext{
*Corresponding Author: Yuan-Xia Fu: Department of Science, Bengbu University, Bengbu 233030, China; Anhui Province Key Laboratory of Optoelectronic Materials Science and Technology, Anhui Normal University, Wuhu 241000, China; Email: fuyuanxia81@aliyun.com

Li Wang, Li Xu: Department of Science, Bengbu University, Bengbu 233030, China
}

əopen Access. () 2020 Y.-X. Fu et al., published by De Gruyter. (cc) BY License undergoes multiphoton ionization to generate free electrons, and then avalanche ionization will occur to produce a large amount of plasma [1]. The specific manifestation is that sparks and noises are present on the surface of the sample. The sample is then analyzed according to the emission spectrum of the plasma. Solids, liquids, and gases, regardless of the shape of the object, can be analyzed by laser-induced plasma spectroscopy technology which can be operated in real time, online, and remotely and is convenient to operate. At present, this technology has been applied to the identification of historical and cultural artifacts, space exploration, industrial inspection, and chemical analysis. Numerous small online inspection systems based on this technology have also been developed [2-8].

In recent years, laser-induced plasma spectroscopy has been used to study trace elements, including qualitative and quantitative analyses of heavy metal elements, research of various processing algorithms, design of multifunctional experiments, and enhancement of experimental devices. Zhang et al. from the Institute of Modern Physics of Chinese Academy of Sciences analyzed the trace elements in apples, strawberries, and kiwifruit using laserinduced plasma spectroscopy [9]. Li (2011) of Jiangxi Agricultural University analyzed the metal elements in the pericarp and pulp of mandarin oranges and navel oranges through laser-induced plasma spectroscopy [10]. Zhang Xu et al. (2012) of Jiangxi Agricultural University used laserinduced plasma spectroscopy to quantitatively analyze the chromium content in apples quantitatively [11]. Kuoray et al. (2013) of Shanxi Agricultural University applied laserinduced plasma spectroscopy to determine the mineral content of three jujube species from different production areas [12]. Abdul Jabbar et al. (2019) of Milpur University of Science and Technology used laser-induced plasma spectroscopy to examine the elemental composition of roots, stems, seeds, and other parts of rice [13]. Given the inevitable interaction between a laser and matter, precise control cannot be carried out through the sample preparation. Consequently, the shock wave generated by the rapid collision between laser and plasma, plasma and plasma, and the interaction between plasma and ambient gas increased the signal uncertainty of the spectral measure- 
ment system and decreased the accuracy of repeatability. Under the influence of the matrix effect, the measurement error was relatively large. These factors have limited the large-scale commercial application of laser-induced plasma spectroscopy. In this paper, laser-induced plasma spectroscopy was used to study the electron temperature changes of the Fe plasma, with time delay, in three kinds of fruit, namely, banana, pineapple, and kiwi fruit are taken as research objects, and the electron temperature changes of Fe plasma in laser-induced fruits with time delay are studied. Plasma electron temperature is an important characteristic of plasma. The experimental results can help us to explore the mechanism and process of the interaction between the laser and matter. In addition, the results can also contribute to laser-induced plasma spectroscopy technology in the analysis of fruits and other crops.

\section{Experimental setup}

A Nd:YAG laser was focused on the surface of the sample through a lens, and plasma is formed on the sample. The emission spectrum of the plasma is received by an optical receiver and transmitted to a spectrometer through an optical fiber. The spectrometer splits the collected spectrum. Then, the ICCD (Intensified Charge-coupled Device) captures the spectrum and converts the collected optical signal into an electrical signal. Finally, it transmits the information to a computer for display. The experimental apparatus is shown in Figure 1. The exposure time was set to $0.2 \mathrm{~ms}$ in the experiment, and each picture is obtained by averaging over 200 laser pulses.

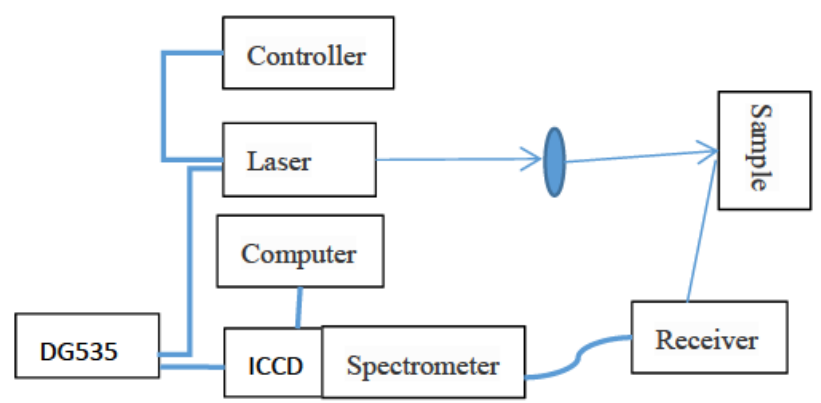

Figure 1: Experimental setup

\section{Spectral analysis}

\subsection{Spectra of fruits with changing gate width}

With a fixed delay and changing the acquisition gate width of ICCD, the 3D spectra of bananas, pineapples, and kiwifruit obtained are, shown in Figure 2, Figure 3, and Figure 4, respectively.

\subsection{Spectra of fruits with changing delay}

With a fixed the acquisition gate width and changing acquisition delay of ICCD, the spectral 3D images of bananas, pineapples, and kiwifruit are shown in Figure 5, Figure 6, and Figure 7, respectively.

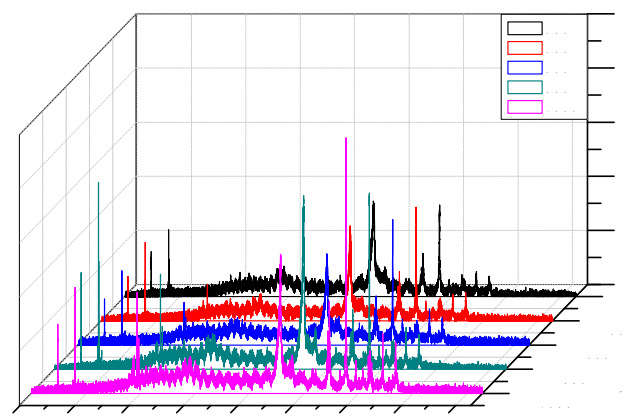

Figure 2: Spectra of a banana with changing gate width

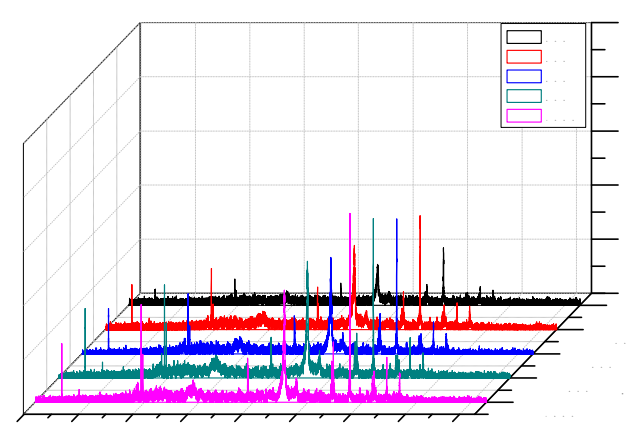

Figure 3: Spectra of a pineapple with changing gate width 


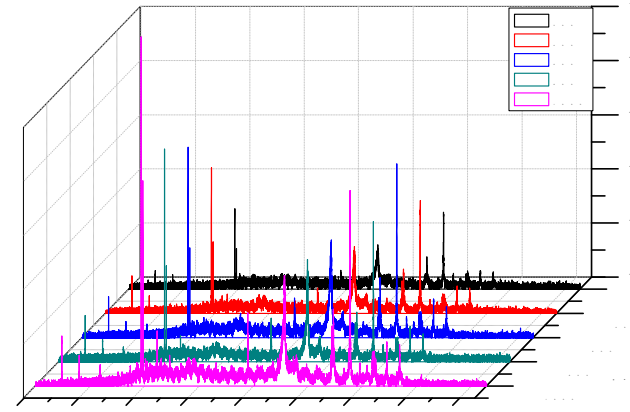

Figure 4: Spectra of a kiwifruit with changing gate width

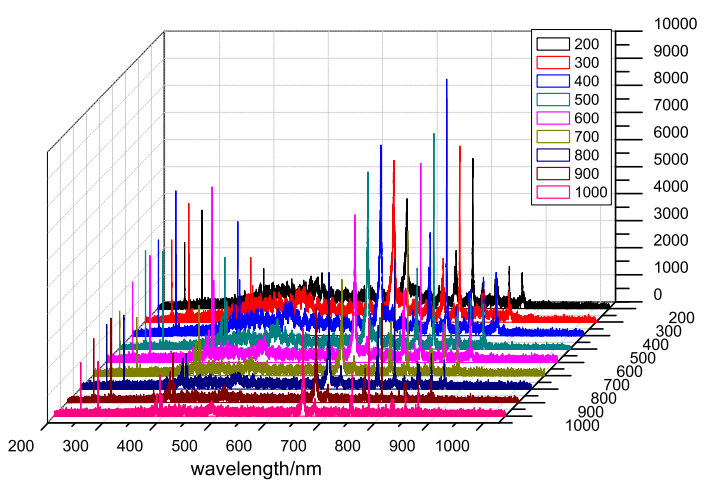

Figure 5: Spectra of a banana with changing delay

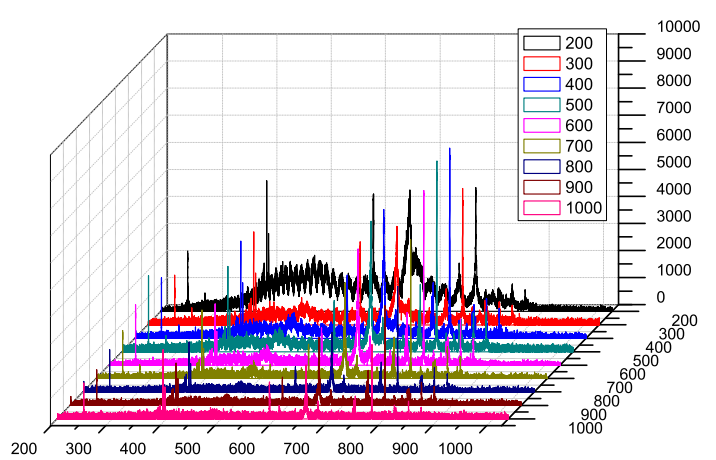

Figure 6: Spectra of a pineapple with changing delay

\subsection{Signal to noise ratio analysis}

To calculate the signal-to-noise ratio, the $696.6 \mathrm{~nm}$ spectral line is selected, and the best optimization conditions

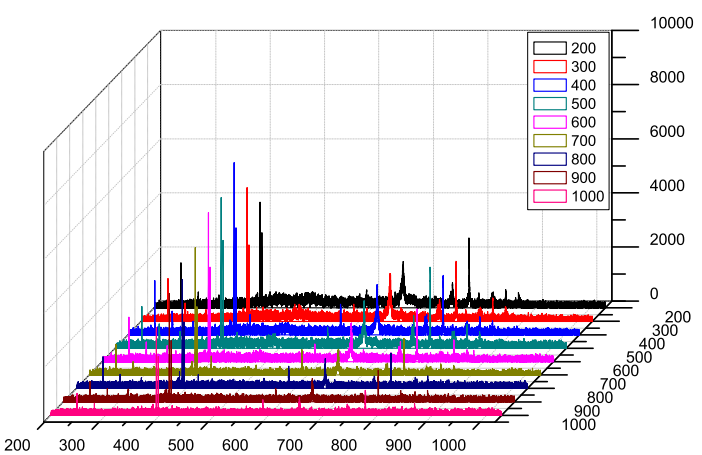

Figure 7: Spectra of a kiwifruit with changing delay

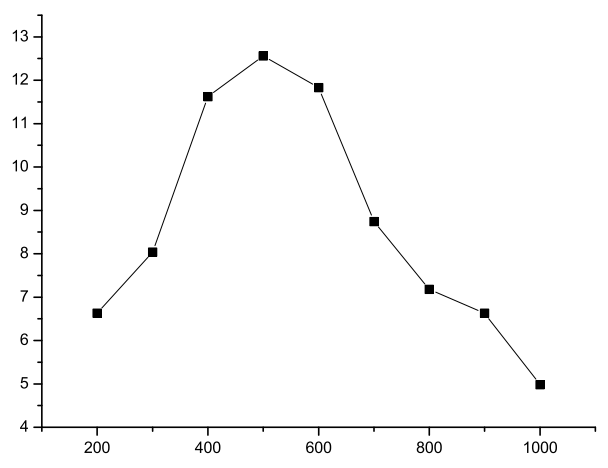

Figure 8: SNR of a banana with different delays

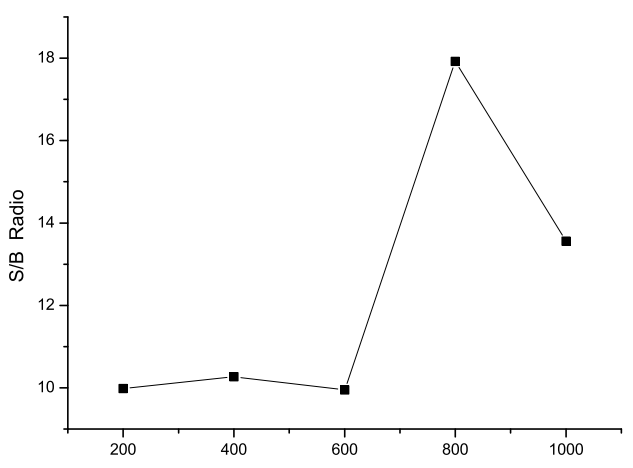

Figure 9: SNR of a banana with different gate widths

of the three fruits can be obtained by analyzing their SNR charts with different delays and gate widths. Firstly, Figure 8 and Figure 9 show that the optimal banana optimization conditions are a delay of $500 \mathrm{~ns}$ and a gate width of 


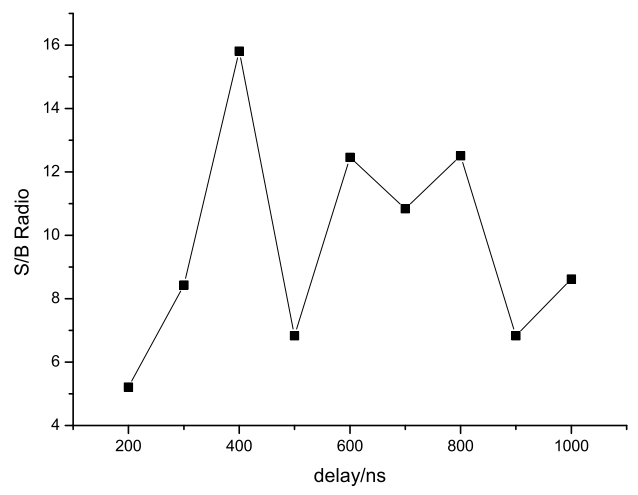

Figure 10: SNR of a pineapple with different delays

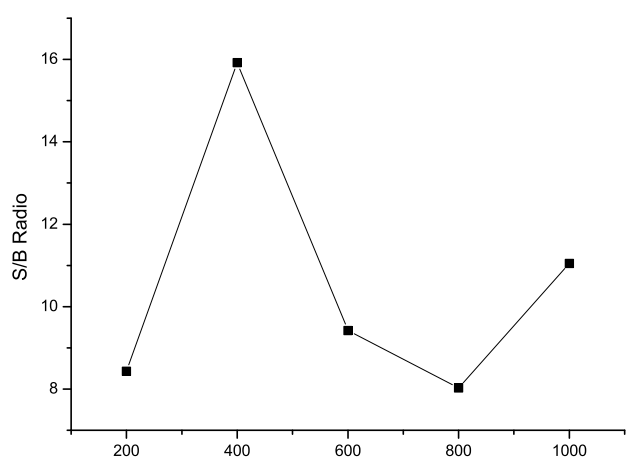

Figure 11: SNR of a pineapple with different gate widths

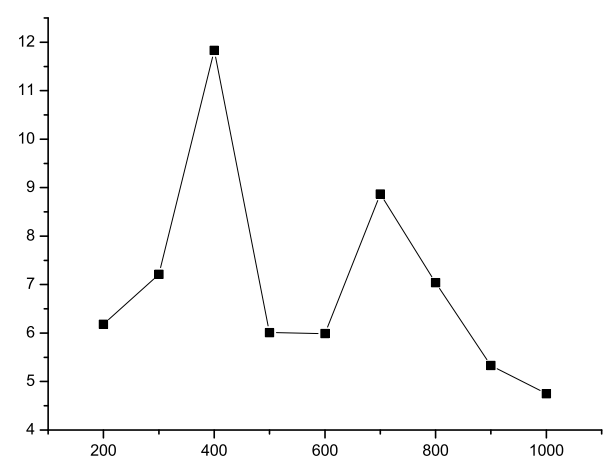

Figure 12: SNR of a kiwi fruit with different delays

800 ns. Secondly, Figure 10 and Figure 11 demonstrate that the optimal optimization conditions for pineapple are a delay of 400 ns and a gate width of 400 ns. Finally, Figure 12 and Figure 13 indicate that the best optimization condi-

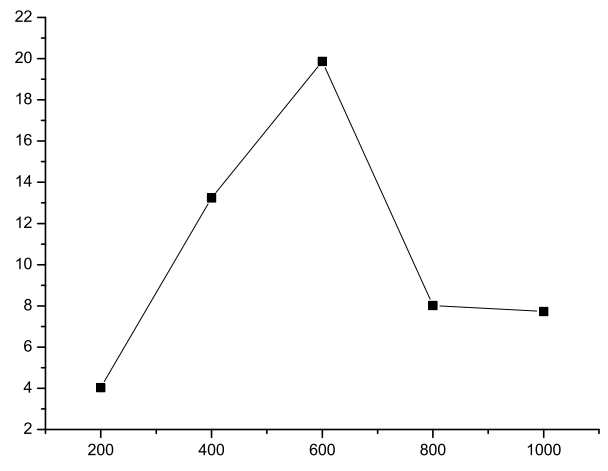

Figure 13: SNR of a kiwi fruit with different gate widths

tions for the kiwifruit are a delay of 400 ns of delay and a gate width of $600 \mathrm{~ns}$.

Given that the exposure delay of ICCD is constant, the time-resolved spectra of different ICCD gate widths are explored. If the gate width is too large despite numerous spectral line signals, several background signals are present. However, if the gate width is too small, the detected signal is weak [13].

When the plasma is initially formed by laser induction, the bremsstrahlung from inside the plasma produces a continuous strong background spectrum, and the characteristic spectral lines of the sample are masked. Later, the background spectral lines decline faster than atomic spectral lines and ion spectral lines. Thus, the emission spectral lines of elements are displayed, and the signalto-noise ratio of the spectral lines increases. If the delay is too small, the spectral lines generated by the laser cannot be filtered out, thus affecting the experimental analysis. When the exposure delay is too large, numerous interference signals enter the spectrometer; thus, choosing the correct exposure delay for spectrogram analysis is especially important [13].

\subsection{Optimum spectrogram}

By analyzing the signal-to-noise ratio of the three different fruits, changing the fixed gate width and delay, and determining the best optimization conditions, the best spectrograms of bananas, pineapples, and kiwifruit can be obtained. 


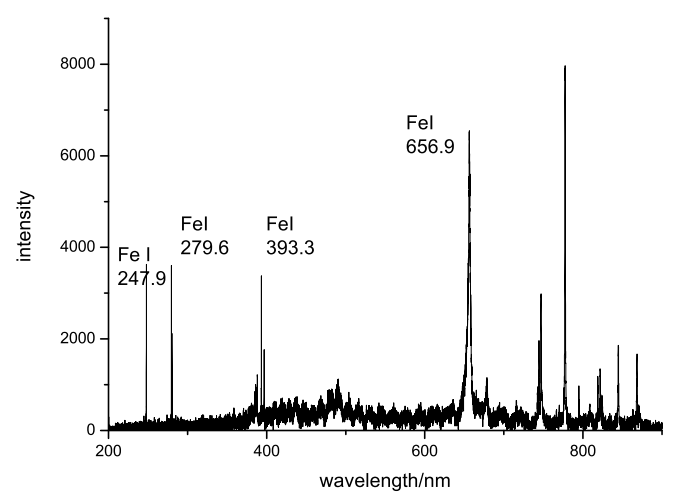

Figure 14: Optimum spectrogram of a banana

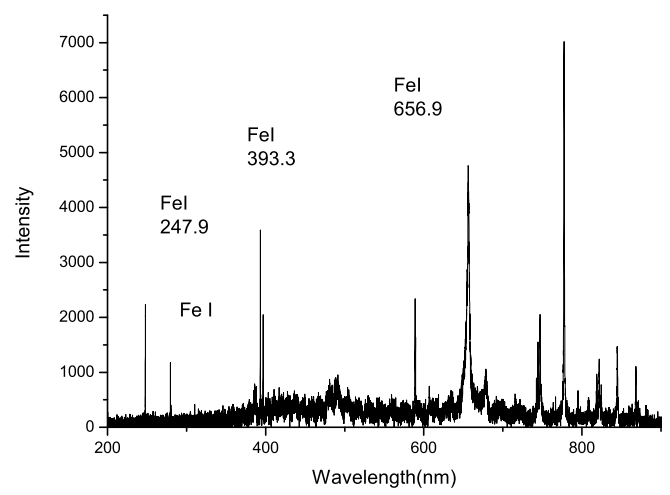

Figure 15: Optimum spectrogram of a pineapple

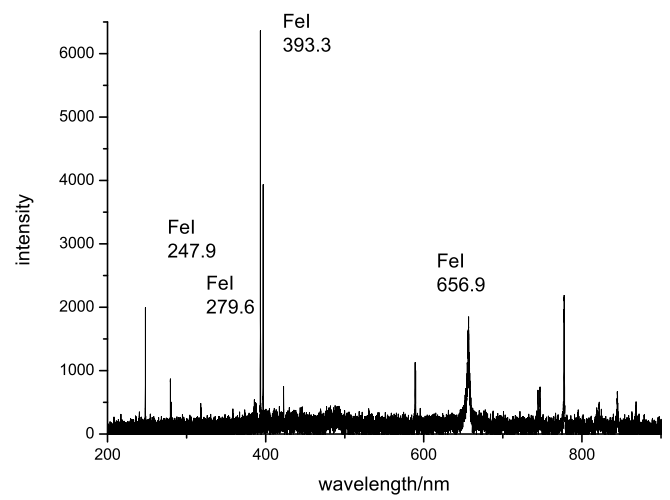

Figure 16: Optimum spectrogram of a kiwi fruit

\section{Electron temperature analysis of the plasma}

The Boltzmann oblique line method is used to calculate the electron temperature of the plasma.

$$
\ln \left(\frac{I M \lambda_{m}}{g_{m} A_{m}}\right)=-\frac{E_{m}}{k_{B} T_{m}}+C .
$$

In Eq. (1), $I_{m}$ is the intensity of the characteristic spectral line, $E_{m}$ is the energy of the upper level, $A_{m}$ is the transition probability of the corresponding spectral line, $g_{m}$ is the statistical weight of the upper levels, and $k_{B}$ is the Boltzmann constant. If $E_{m}$ is the abscissa and $\ln \left(\frac{I M \lambda_{m}}{g_{m} A_{m}}\right)$ is the ordinate, then the fitting slope $\frac{1}{k_{B} T_{m}}$ is obtained by linear fitting. Finally the electron temperature $\left(T_{m}\right)$ can be obtained by calculation. Among them, the relative intensity $\left(I_{m}\right)$ must be obtained using Lorentz fitting [15-18]. The Boltzmann oblique line method in calculating the electron temperature of plasma is very convenient, but it can only use atomic lines or ion lines with the same valence of the same elements. When the excitation energies of the selected spectral lines are close, the calculation error is large, then we can add different valence ion lines of the same element with the Boltzmann multiline method [18].

\subsection{Electron temperature of laser-induced Fe plasma in bananas}

For the calculation of the electron temperature of the $\mathrm{Fe}$ plasma in fruits, four spectral lines of Fe with wavelengths of $247.9 \mathrm{~nm}, 279.6 \mathrm{~nm}, 393.3 \mathrm{~nm}$, and $656.9 \mathrm{~nm}$ were selected. Table 1 lists the relevant spectral constants of the four spectral lines.

The Boltzmann oblique lines $\left(\ln (I \lambda / g A) \sim E_{k}\right)$ of three spectral lines are calculated. Then, the electron temperature of Fe plasma can be obtained by slope.

The relationship between the electron temperature characteristics of the laser-induced Fe plasma in a banana and the time delay is shown in Figure 18. In the time delay range of 200-300ns, the electron temperature of the Fe plasma increases as the delay increases. In the delay range of 300-1000ns, the electron temperature of the $\mathrm{Fe}$ plasma decreases as the delay increases, reaching a maximum value at $300 \mathrm{~ns}$, and the electron temperature varies between $12388.29 \mathrm{~K}$ and $19958.3 \mathrm{~K}$. 
Table 1: Excitation energy, statistical weight of the upper levels of the Fe line, and the corresponding transition probability for the selected spectral lines

\begin{tabular}{ccccc}
\hline Wavelength $(\mathrm{nm})$ & Excitation energy $\left(\mathrm{cm}^{-1}\right)$ & Excitation energy $(\mathrm{eV})$ & $\mathrm{g}_{k}$ & $\mathrm{~A}_{k}\left(10^{6} \mathrm{~s}^{-1}\right)$ \\
\hline 247.9 & 48304.643 & 5.978 & 5 & 21 \\
\hline 279.6 & 77861.650 & 9.636 & 10 & 20.00 \\
393.3 & 50186.834 & 6.211 & 5 & 5.92 \\
656.9 & 53393.673 & 6.608 & 9 & 6.00 \\
\hline
\end{tabular}

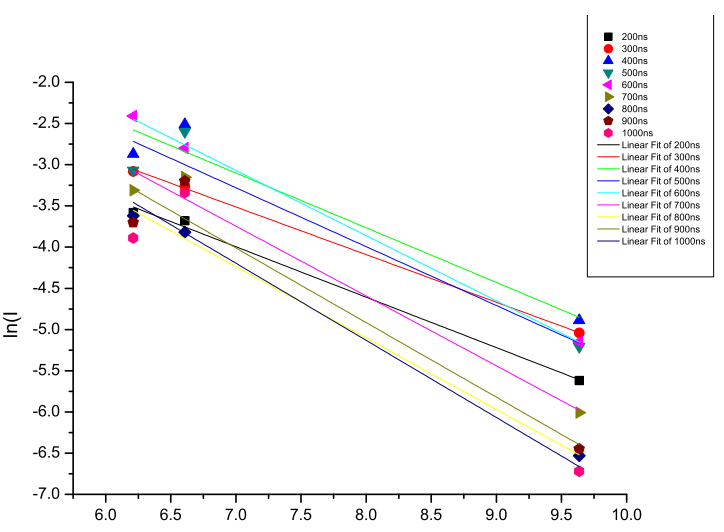

Figure 17: Boltzmann oblique lines of a banana with different delays

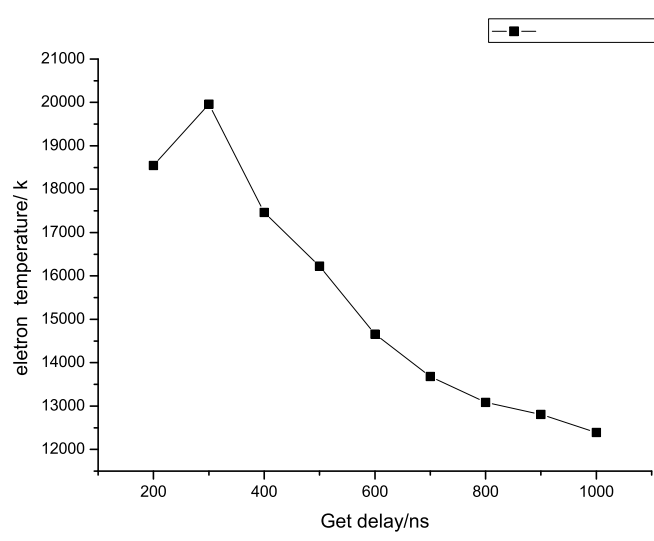

Figure 18: Temporal evolution of the electron temperature of the laser-induced Fe plasma in a banana

\subsection{Electron temperature of laser-induced Fe plasma in pineapples}

For the calculation of the electron temperature of the Fe plasma in pineapples, three spectral lines with Fe wavelengths of $279.6 \mathrm{~nm}, 393.3 \mathrm{~nm}$, and $656.9 \mathrm{~nm}$ were selected. Table 1 lists the relevant spectral constants of the three spectral lines. The Boltzmann oblique lines $(\ln (I \lambda / g A) \sim$ $E_{k}$ ) of the three spectral lines were calculated. Then, the

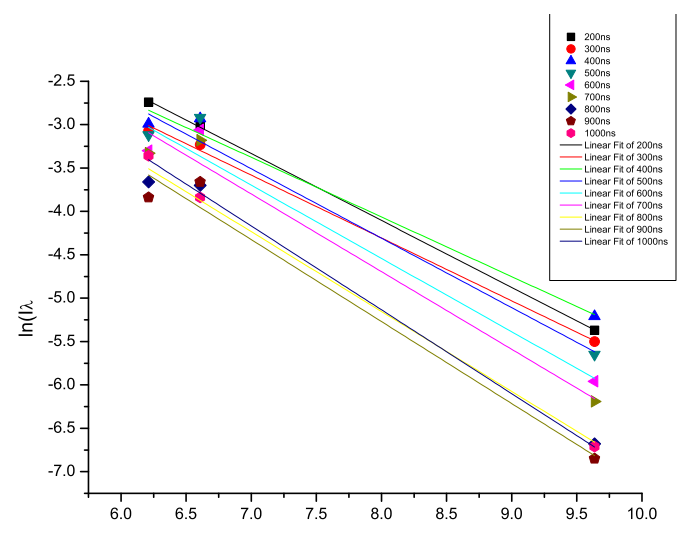

Figure 19: Boltzmann oblique lines of a pineapple with different delays

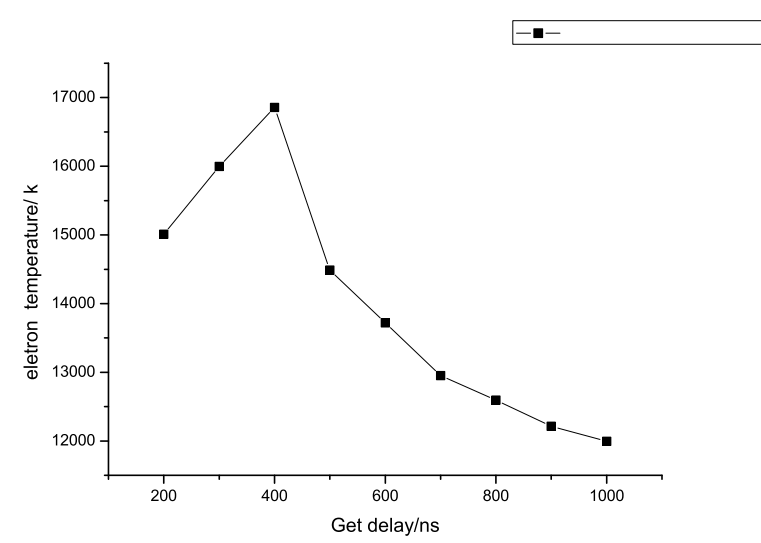

Figure 20: Temporal evolution of the electron temperature of laserinduced Fe plasma in a pineapple

electron temperature of the Fe plasma was obtained from the slope.

The relationship between the electron temperature characteristics of the laser-induced Fe plasma in pineapples and the time delay is shown in Figure 20. In the time delay range of 200-400 ns, the electron temperature of the Fe plasma decreases as time delay increases. In the time delay range of $400-1000 \mathrm{~ns}$, the electron temperature 


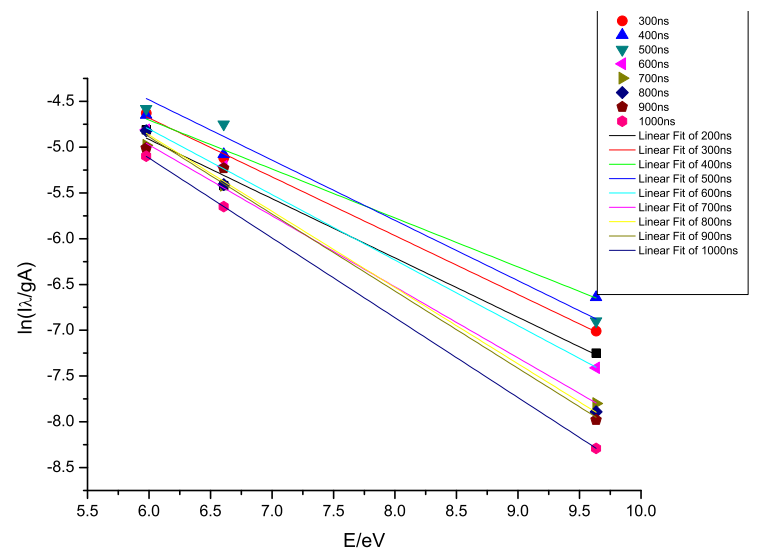

Figure 21: Boltzmann oblique lines of a kiwi fruit with different delays

of the Fe plasma decreases as time delay increases after reaching the maximum value at $400 \mathrm{~ns}$. The electron temperature varies between $11994.21 \mathrm{~K}$ and $16856.4 \mathrm{~K}$.

\subsection{Electron temperature of laser-induced Fe plasma in kiwi fruit}

For the calculation of the electron temperature of the $\mathrm{Fe}$ plasma in kiwifruit, three spectral lines with Fe wavelengths of $247.9 \mathrm{~nm}, 279.6 \mathrm{~nm}$, and $656.9 \mathrm{~nm}$ were selected. Table 1 lists the relevant spectral constants of the three spectral lines. The Boltzmann oblique lines $(\ln (I \lambda / g A) \sim$ $E_{k}$ ) of the three spectral lines are calculated. Then, the electron temperature of the Fe plasma was obtained from the slope.

The relationship between the electron temperature characteristics of the Fe plasma in a laser-induced kiwifruit and the time delay is shown in Figure 22. In the time delay range of 200-400 ns, the electron temperature of the Fe plasma decreases as time delay increases. In the time delay range of 400-1000 ns, the electron temperature decreases with the increase of the time delay after reaching the maximum value at $400 \mathrm{~ns}$. Moreover, the electron temperature varies between $13388.2 \mathrm{~K}$ and $19607.54 \mathrm{~K}$.

According to the analysis of the evolution characteristics of the plasma electron temperature of three fruits with time delay, the plasma electron temperature gradually increases. After rising to the maximum value, the plasma electron temperature then decreases with increasing time delay, and the speed decelerates. The plasma diffuses outward after it is formed, and its diffused kinetic energy is converted from thermal energy. Thus, the plasma electron temperature decreases with the expansion of volume.

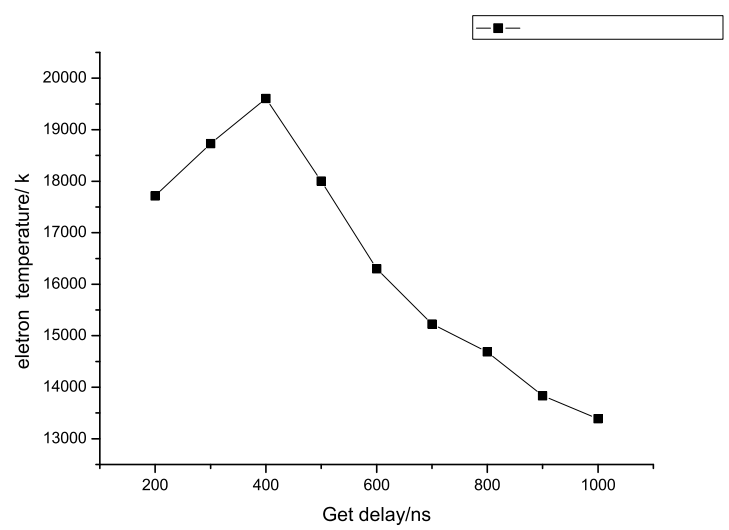

Figure 22: Temporal evolution of the electron temperature of laserinduced Fe plasma in a kiwifruit

When the plasma electron temperature falls to a lower range, the efficiency of thermal energy conversion to kinetic energy decreases, and the downward trend of the plasma electron temperature slows down.

The time evolution trends are similar, but the electron temperatures are different at the same time delay. The highest electron temperatures are different, and the delay corresponding to the maximum value are also different.

\section{Conclusion}

In this paper, the spectra of bananas, pineapples, and kiwifruits are measured for a series of experiments, and the electron temperature characteristics of the Fe plasma induced by a laser are analyzed with time delay. The range of the electron temperatures of the Fe plasma in bananas, pineapples, and kiwi fruits was 12388.2919958.3 K, 11994.21-16856.4 K, and 13388.2-19607.32 K, respectively.Laser-induced plasma spectroscopy has attracted much attention due to its unique advantages. For example, samples can be liquid, solid, or gaseous and it allows for long-range monitoring, especially under special circumstances. In addition, the analysis time is short, and the process can simultaneously detect multiple elements on line. It is possible to combine laser-induced plasma technology, Raman spectrum technology, and fluorescence spectrum technology to obtain comprehensive material composition information. Although certain problems in the practical application of laser-induced plasma spectroscopy still exist, the field of application of this technology is expected to expand and become popular in the future along with the development of science. 
Acknowledgement: The National Natural Science Foundation of China(11604003), the Key Program of Natural Science Foundation of Anhui Province (KJ2019A085) and the Anhui Province Key Laboratory of Optoelectronic Materials Science and Technology (OMST201703)

\section{References}

[1] Lu TX. Principle and application of laser spectroscopy. Hefei: China University of Science and Technology Press; 2009. pp. 201-5.

[2] Xu XB, Du CW, Ma F, Shen Y, Wu K, Liang D, et al. Detection of soil organic matter from laser-induced breakdown spectroscopy (LIBS) and mid-infrared spectroscopy (FTIR-ATR) coupled with multivariate techniques. Geoderma. 2019;355(1):113905.

[3] Tang HJ, Hao XJ, Hu XY, et al. Study on spectral time evolution of laser induced Cu plasma. Laser and Infrared. 2018;48(11):13415.

[4] Ye TB. Experimental study on laser plasma shielding. Nanjing: Nanjing University of Technology; 2007.

[5] Giorgio S. Recent advances and future trends in LIBS applications to agricultural materials and their food derivatives: an overview of developments in the last decade (2010-2019). Part II. Crop plants and their food derivatives. Trends Analyt Chem. 2019;118:453-69.

[6] Hou GY, Wang P, Tong CZ. Progress in laser-induced breakdown spectroscopy and its applications. China Optics. 2013;6(4):4912.

[7] Zhang GY, Ji H, Li ST, Zheng HM. Characterization of plasma induced by laser effect on coal sample. Guang Pu Xue Yu Guang Pu Fen Xi. 2016 May;36(5):1323-7.
[8] Meng DS, Zhao NJ, Ma MJ, Gu YH, Yu Y, Fang L, et al. Rapid soil classification with laser induced breakdown spectroscopy. Guang Pu Xue Yu Guang Pu Fen Xi. 2017 Jan;37(1):241-6.

[9] Zhang DC, Ma XW, Zhu XL, et al. Application of laser-induced breakdown spectroscopy in analyzing microelements in three kinds of fruit samples. Wuli Xuebao. 2008;57(10):6348-9.

[10] Li QL. Analysis and research on laser induced breakdown spectroscopy applied to determination of metal elements in citrus and soil. Nanchang: Jiangxi Agricultural University; 2011.

[11] Zhang X, Yao MY, Liu MH, et al. Quantitative analysis of chromium in apples by laser-induced breakdown spectroscopy. Laser and Infrared. 2012;4(5):495-6.

[12] Guo R, Wang XY. Determination of mineral elements in red jujube with laser-induced breakdown spectroscopy. Shanxi Nongye Daxue Xuebao. 2013;33(6):498-9.

[13] Jabbar A, Akhtar M, Ali A, Mehmood S, Iftikhar S, Baig MA. Elemental composition of rice using calibration free laser induced breakdown spectroscopy. Optoelectron Lett. 2019;15(1):53-63.

[14] Fu YX, Tang YQ, Xu L, et al. Measurement on trace element composition of Chinese medicinal materials by laser induced breakdown spectroscopy. Journal of Bengbu University. 2017;6(3):204.

[15] Wang L, Fu YX, Xu L, et al. The Effect of Sample temperature on characteristic parameters of the nanosecond laser-induced $\mathrm{Cu}$ Plasma. spectroscopy and spectral analysis. 2019; 39(4): 12471251.

[16] Fu YX, Wang L, Ma LY, et al. An investigation on the laser induced Cu plasma characteristics. Journal of Atomic and Molecular Physics. 2019;36(02):263-7.

[17] Wang L, Zhou Y, Fu YX, Xu L, Gong H, Cheng R. Effect of sample temperature on radiation characteristics of nanosecond laseinduced soil plasma. Chin J Chem Phys. 2019;32(06):760-4.

[18] Yu JL, Li C, Yao GX, et al. Spatial Evolution Characteristics of Laser-induced Plasma in Liquid Matrix. Chin J Lasers. 2019;46(8):0802001. 